\title{
HRI'16 Chairs' Welcome
}

We are excited to welcome you to the Eleventh Annual ACM/IEEE International Conference on HumanRobot Interaction (HRI 2016). The HRI Conference is a highly selective, single track, international meeting showcasing the best research in human-robot interaction, with roots in and broad participation from various communities of scholars, including but not limited to robotics, human-computer interaction, artificial intelligence, engineering, social and behavioral sciences, and design.

The theme of this year's conference is "Natural Interaction," an increasingly important research focus and design goal as robotic technologies become incorporated into everyday life. Achieving natural humanrobot interaction requires the input of many technical, social, and design disciplines, as well as reflection on the societal consequences and ethical significance of robotic development. To represent the multitude of disciplines and approaches that contribute to this work and the HRI field more generally in the conference program, we solicited and reviewed papers under four submission themes: "Studies of HRI", "Technical Advancements in HRI", "HRI Design", and "Theory and Method in HRI." Each submission theme was overseen by a dedicated theme chair and reviewed by a dedicated group of program committee members, who worked together with the program chairs to define and apply review criteria appropriate to each of the four contribution types.

The conference attracted 181 submissions from Asia-Pacific, Europe, the Middle East, and North America. Each full paper was aligned with a theme-appropriate subcommittee, and subsequently reviewed through a double-blind process, which was followed by a rebuttal phase, and shepherding where suggested by the program committee. As a result of the review process, the program committee selected $45(24.8 \%)$ of the submissions for presentation as full papers at the conference. As the conference is jointly sponsored by IEEE and ACM, papers are archived in both IEEE Xplore and the ACM Digital Library.

Along with the full papers, the conference program and proceedings include the Late Breaking Reports, Videos, Demos, and a new Alt-HRI section. Out of 95 total submissions, 71 (74.7\%) Late Breaking Reports were accepted and will be presented as posters at the conference. Eleven (85\%) short videos were accepted for presentation during a dedicated video session from 13 submissions. The program also includes four demos of robot systems that participants will have an opportunity to interact with during the conference. We're very excited to introduce an Alt-HRI session in this year's program, consisting of five papers (selected out of 11 submissions) that push the boundaries of thought and practice in the field. We

Invited authors from the Journal of HRI will also orally present their work. Finally, we have the pleasure of presenting three inspiring keynote speakers who will discuss topics relevant to HRI: Dr. Aude Billard from EPFL in Switzerland, Dr. Marc Sagar from the University of Auckland in New Zealand, and Dr. Robert Sparrow from Monash University in Australia. 
HRI 2016 was made possible through the significant volunteer efforts of the organizing committee, program committee, reviewers and the Steering Committee. We thank the keynote speakers, financial supporters Honda and Aldebaran, and international reviewers for their support and participation. The conference is sponsored by ACM SIGCHI, ACM SIGART, IEEE Robotics and Automation Society, and is in cooperation with AAAI and HFES.

We also want to commend and thank the authors who submitted papers, videos, and demos to HRI 2016, and whose efforts make HRI an exciting field and vibrant community. We hope you will enjoy the conference content, seeing old friends and making new ones, and your visit to Christchurch.

\section{Christoph Bartneck}

HRI'16 General Co-Chair

University of Canterbury, New Zealand
Yukie Nagai

HRI'16 General Co-Chair

Osaka University, Japan

\section{Ana Paiva}

HRI'16 Program Co-Chair

IST, University of Lisbon, Portugal

\section{Selma Šabanović}

HRI'16 Program Co-Chair

Indiana University, USA 